\title{
An Adaptive Laplacian Based Interpolation Algorithm for Noise Reduction in Body Surface Potential Maps
}

\author{
Ali S Rababah, Dewar D Finlay, Daniel Guldenring, Raymond Bond, James D McLaughlin \\ Ulster University, Belfast, Northern Ireland
}

\begin{abstract}
Body surface potential maps (BSPMs) are typically recorded from a large number of ECG leads that cover the entire thorax. This improves diagnostic accuracy and is required in Electrocardiographic imaging (ECGi). BSPMs recorded in the clinical setting may have some leads that are noisy due to poor skin electrode contact.

We analyzed 117 lead BSPMs recorded from 360 subjects. We successively simulated the removal of ECG leads at various locations and tested the ability of our algorithm to accurately reconstruct the missing information.

When seven electrodes were removed, the algorithm could reconstruct BSPM patterns from QRS segments with median RMSE of $6.24 \mu \mathrm{V}$ and $12.15 \mu \mathrm{V}$ and $C C$ of 0.999 and 0.997 when Laplacian method and PCA based method were used respectively.

This work shows that noisy BSPM leads, which often manifest in the clinical setting, can be more accurately reconstructed using our Laplacian based interpolation algorithm, when low number of missed electrodes in regions where electrodes are organised in a well distributed and tight mesh.
\end{abstract}

\section{Introduction}

Cardiovascular diseases are the leading cause of death, disability, and impaired quality of life. According to the American Heart Association (AHA), cardiovascular diseases were responsible for 17.9 million deaths in 2015 alone. This number is expected to grow in the following years [1]. Since the standard 12-lead ECG is a cheap, fast, and non-invasive clinical tool, it has been the most common method in the clinical practice to noninvasively assess cardiac activity and diagnose cardiac diseases including infarction, ischemia, abnormalities of conduction, hypertrophy, and cardiomyopathy. However, low spatial resolution attributed to using only 10 electrodes causes a loss of important diagnostic information and limits its usability as an accurate and specific diagnostic tool. This motivated scientists and researchers to develop Body surface potential mapping (BSPM) systems which record the electrical activity of the heart from 32 to 300 electrodes attached to body torso. This increased spatial sampling provides a more complete picture of the heart electrical activity as projected to the body surface and improves the sensitivity and specificity in detecting and characterizing heart diseases [2]. Another motivation for BSPMs is to utilize it together with torso and heart geometries and conductivities to estimate the epicardial potentials on the heart surface. In other words, BSPM is an essential part of ECG Imaging (ECGI) system which has demonstrated feasibility in estimating potentials, electrograms, isochrones and repolarization patterns on the heart surface [3].

One the challenges of BSPM recording is the management of the large number of recording leads/electrodes during the acquisition process. And, often BSPMs recorded in the clinical setting may have some leads that are noisy due to issues such as poor skin electrode contact. In this study, we will investigate the performance of two interpolation methods including a Laplacian method and a Principal Component Analysis based method in interpolating noise contaminated BSPM leads and in reconstruction of BSPM potential distributions.

\section{Methods}

We analyzed 117 lead BSPMs recorded from 360 subjects $($ Normal $=120, \mathrm{MI}=120, \mathrm{LVH}=120)$. The recording apparatus and procedure has previously been described in $[4,5]$. The analysis was performed on QRS map frames. We successively simulated the removal of ECG leads at various locations and tested the ability of our algorithm to accurately reconstruct the missing information. The reason behind removing from $1-7$ electrodes is to mimic what we expect to face in clinical practice when up to one whole strip is missed.

Two methods of interpolation were used; Laplacian interpolation [6] and Principal Component Analysis [7]. Laplacian Interpolation is an efficient method for 
restoring missing data on a grid. The value at missed electrode is approximated by calculating the weighted average of the values at its direct neighbors. the weighting coefficients are calculated according to the distance between vertices on the triangulated mesh of body torso [6]. PCA based interpolation was performed as follow; firstly, a training set of BSPM data of 400 patients were used to calculate the first 15 principal components (PCs) which accounts for 98.91 per cent of total variability in the data. Then, these 15 PCs were exploited to estimate the missing information in the BSPM test data.

Following to estimating the missing electrodes using previously described methods, the Actual and interpolated BSPM distributions were compared using two independent and widely used metrics; RMS error (RMSE) and correlation coefficient (CC) defined as:

$$
R M S E=\sqrt{\frac{\sum_{i=1}^{n}\left(x_{i}-y_{i}\right)^{2}}{n}}
$$

Where $x_{i}$ and $y_{i}$ are the ith sample from a measured and estimated BSPMs respectively, and $\mathrm{n}$ is the number of samples.

$$
C C=\frac{n\left(\sum x y\right)-\left(\sum x\right)\left(\sum y\right)}{\sqrt{\left.\left[n \sum x^{2}-\left(\sum x\right)^{2}\right]\left[n \sum y^{2}\right]-\left(\sum y\right)^{2}\right]}}
$$

Where $\mathrm{x}, \mathrm{y}$ are the measured and estimated BSPMs respectively, and $\mathrm{n}$ is the number of samples.

Computational routines were implemented in MATLAB (The MathWorks Inc, Natick, MA) for processing and analysis of data. MAP3D visualization software was used for general display and inspection of potential distributions on heart and torso surfaces [8].

\section{Results}

As shown in Table 1 and Table 2 the values of median RMSE increases as the number of interpolated leads increases. A closer look to both tables indicates that Laplacian interpolation showed better reconstruction of missing information in QRS map frames than PCA method. The reason of investigating up to $7 \mathrm{missed}$ electrodes is to simulate the poor contact of one strip that contains 7 electrodes. Figure 3 and Figure 4 better illustrate the range of RMSE and CC values for all patients across all frames. It shows median, interquartile range, and whiskers from minimum to maximum. This means that Laplacian interpolation reconstructed 90 per cent of BSPMs with approximately $30 \mu \mathrm{V}$ RMSE and $0.994 \mathrm{CC}$ when up to seven electrodes were missed.

Table 1. Median RMSE and Median CC when using
Laplacian interpolation method to reconstruct QRS map

\begin{tabular}{|c|c|c|c|}
\hline $\begin{array}{l}\text { Number of } \\
\text { interpolated } \\
\text { electrodes }\end{array}$ & $\begin{array}{l}\text { Median } \\
(\mu \mathrm{V})\end{array}$ & RMSE & Median CC \\
\hline 1 & 1.09 & & 1 \\
\hline 2 & 2.18 & & 1 \\
\hline 3 & 3.14 & & 1 \\
\hline 4 & 4 & & 1 \\
\hline 5 & 4.79 & & 0.999 \\
\hline 6 & 5.54 & & 0.999 \\
\hline 7 & 6.24 & & 0.999 \\
\hline
\end{tabular}
frames.

Table 2. Median RMSE and Median CC when using Principal component analysis to reconstruct QRS map frames.

\begin{tabular}{llll}
\hline $\begin{array}{l}\text { Number of } \\
\text { interpolated } \\
\text { electrodes }\end{array}$ & $\begin{array}{l}\text { Median } \\
(\mu \mathrm{V})\end{array}$ & RMSE & Median CC \\
\hline 1 & 1.7 & 1 \\
2 & 3.47 & 1 \\
3 & 5.18 & 0.999 \\
4 & 6.91 & 0.999 \\
5 & 8.65 & 0.999 \\
6 & 10.34 & 0.998 \\
7 & 12.15 & 0.997 \\
\hline
\end{tabular}

Figure 1 and Figure 2 show the difference in performance of the two interpolating methods. Both methods faithfully reconstructed the missing potentials and allowed accurate location of the potential minimum and maximum. However, visual examination of both Figures shows that Laplacian interpolation performed much better than PCA in regaining lost information. This appears clearly when examining the contour lines around lead 171.

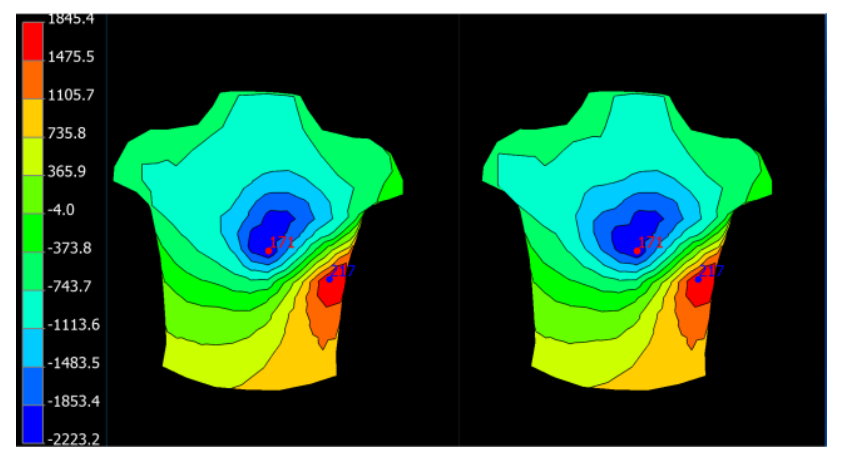

Figure 1. Original (left) and reconstructed (right) BSPM (46ms into the QRS) after performing Laplacian Interpolation $(\mathrm{RMSE}=45.7 \mu \mathrm{V}, \mathrm{CC}=0.999)$. 


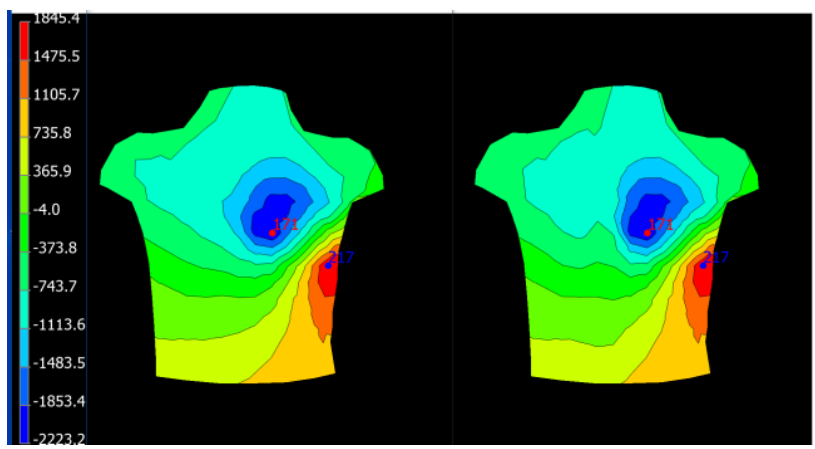

Figure 3. Original (left) and reconstructed (right) BSPM (46ms into the QRS) after interpolating using Principal Component Analysis ( $\mathrm{RMSE}=55.1 \mu \mathrm{V}, \mathrm{CC}=0.998)$. well distributed and tight mesh [9]. A study performed by Laura et al. suggested that Linear interpolation to reconstruct lost BSPMs does not improve, and in some cases further deteriorates reconstruction accuracy [10]. This is the case when interpolating in large region of missing data but does not hold true for low number of missed electrodes in regions where electrodes are organised in a well distributed and tight mesh.

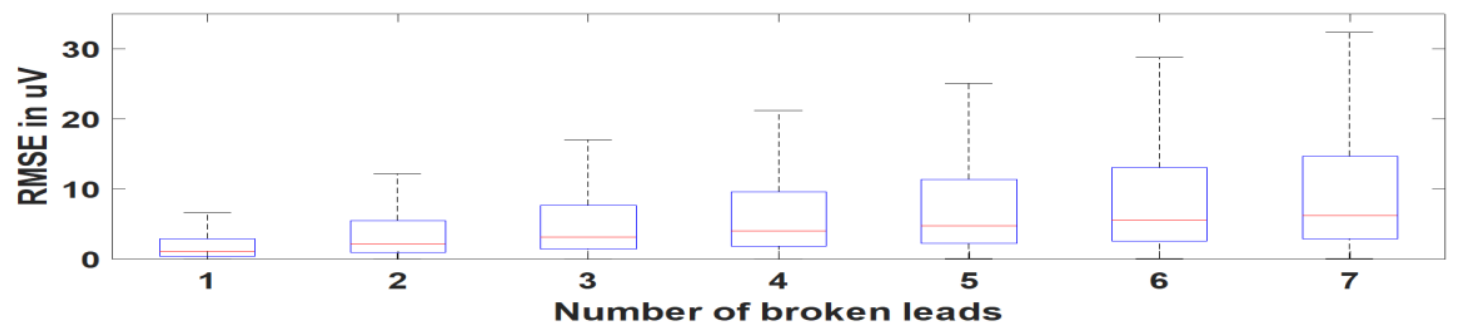

(a)

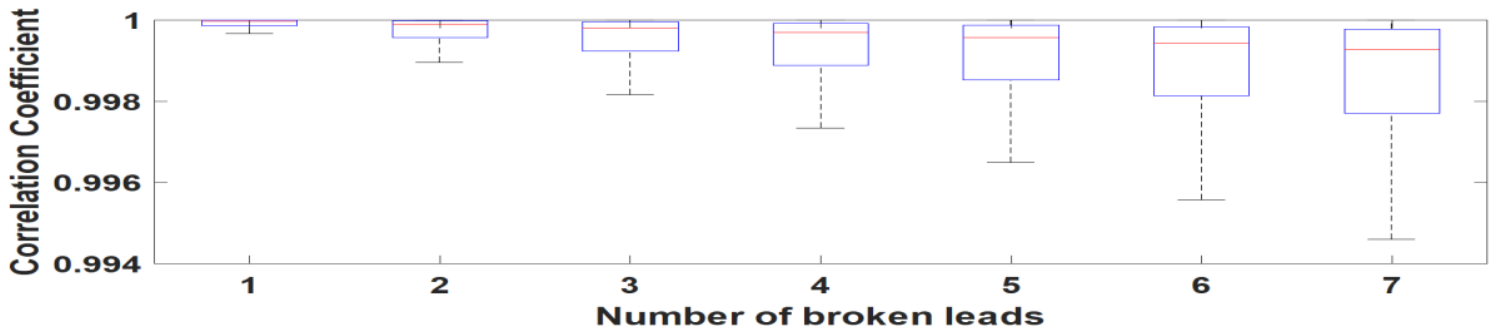

(b)

Figure 2. Performance of Laplacian method in reconstructing missed leads. a) RMSE and b) CC values are shown (median, interquartile range, and whiskers from minimum to maximum.

\section{Discussion}

Our results show that Laplacian interpolation performed better than PCA method in reconstructing lost information in BSPMs. Rudy et al. showed that Laplacian interpolation introduced significant errors to epicardial inverse reconstruction when interpolating potentials in large regions of missing data. However, it did very well in local interpolation between electrodes organized in a

\section{Conclusion}

Laplacian interpolation is a good choice for interpolating BSPMs when the number of missed electrodes is low, and interpolation is needed between electrodes organised in well distributed and tight mesh. 


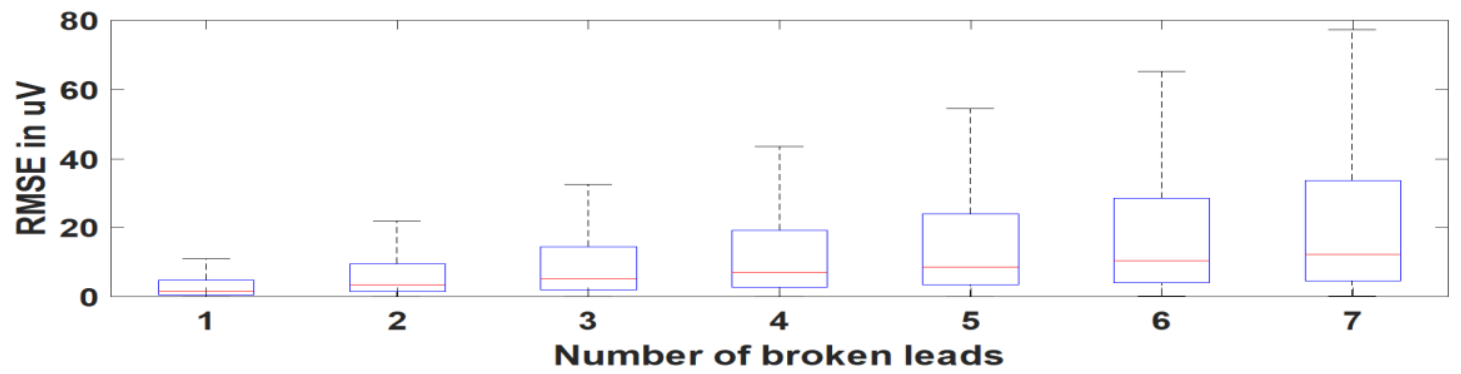

(a)

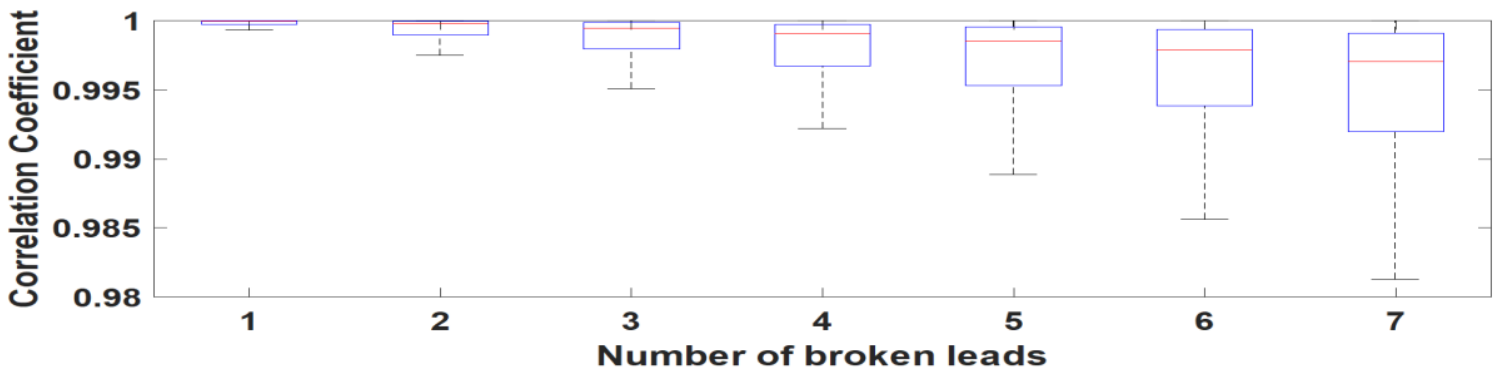

(b)

Figure 4. Performance of PCA based method in reconstructing missed leads. a) RMSE and b) CC values are shown (median, interquartile range, and whiskers from minimum to maximum).

\section{Acknowledgements}

This project is supported by the European Union's INTERREG VA Programme, managed by the Special EU Programmes Body (SEUPB)

\section{References}

[1] Benjamin EJ, Virani SS., Callaway CW, Chamberlain AM, Chang AR, Cheng $\mathrm{S}$ et al. Heart disease and stroke statistics-2018 update: A report from the american heart association. Circulation 2018;137: 67-492.

[2] Macfarlane PW, van Oosterom A, Pahlm O, Kligfield P, Janse M, Camm J. Comprehensive Electrocardiology. Springer London 2010.

[3] Berger T, Hintringer F, Fischer G. Noninvasive imaging of cardiac electrophysiology. Indian Pacing and Electrophysiology Journal. 2007; 7:160-165.

[4] Kornreich F, Montague TJ, Rautaharju PM. Identification of first acute $Q$ wave and non-Q wave myocardial infarction by multivariate analysis of body surface potential maps. Circulation 1991;6:2442-2453.

[5] Montague TJ, Smith ER, Cameron DA, Rautaharju PM, Klassen GA, Felmington CS, et al. Isointegral analysis of body surface maps: surface distribution and temporal variability in normal subjects. Circulation 1981;5:11661172.

[6] Oostendorp TF, van Oosterom A, Huiskamp G. Interpolation on a triangulated 3D surface. J. Comput. Phys. 1989; 80:331-343.
[7] Lux R, Karhunen-loeve representation of ECG data. Journal of Electrocardiology. 1992;25:195 - 198 .

[8] MacLeod RS, Johnson CR. Map3d: interactive scientific visualization for bioengineering data: IEEE Engineering in Medicine and Biology Society 15th Annual International Conference, IEEE Press, New York 1993;30-31

[9] BurnesJE, Kaelber DC, Taccardi B, Lux R L, Ershler PR, Rudy Y. A field-compatible method for interpolating biopotentials. Annals of Biomedical Engineering 1998; 26, 37-47.

[10] Bear L, Potse M, Duchateau J, Zemzemi N, Coudière Y Dubois R, Accuracy of lead removal vs linear interpolation in non-invasive electrocardiographic imaging (ECGI). Computing in Cardiology Conference (CinC). 2015: 941944

Address for correspondence.

Ali Rababah

University of Ulster

Shore Road

Belfast

BT37 0QB

Rababah-A@ulster.ac.uk 